\title{
HYPERBOLIC GENERATORS FOR FUCHSIAN GROUPS ${ }^{1}$
}

\author{
ROBERT J. SIBNER
}

The standard system of generators for a Fuchsian group contains, in general, some elliptic and parabolic transformations. It is not, however, well known that one can always choose a system of generators consisting only of hyperbolic transformations. ${ }^{2}$ We will obtain this result by establishing a stronger statement: there exists a convex noneuclidean fundamental polygon, whose sides are identified by hyperbolic transformations.

Clearly, fundamental polygons other than the "Fricke polygons" are needed. Such polygons are considered in [6] where most of the facts which we shall need are developed. We will of ten omit details and proofs when they would be only slight modifications of those in [6].

1. Preliminaries. A finitely generated discontinuous group $G$ of conformal self-mappings of the unit disc $U$ is called a Fuchsian group. Let $\pi$ denote the canonical mapping of $U$ on to $U / G$. Then $U / G$, with the induced conformal structure, is a Riemann surface. Given in tegers $g, h, n, l$ and $\nu_{1}, \cdots, \nu_{n}$ satisfying

$$
\begin{gathered}
g \geqq 0, \quad h \geqq 0, \quad n \geqq l \geqq 0, \\
1<\nu_{j}<\infty \quad \text { for } 1 \leqq j \leqq l \text { and } v_{j}=\infty \text { for } l+1 \leqq j \leqq n,
\end{gathered}
$$

we will say (following [3]) that $G$ has signature

$$
\left(g, h, n ; \nu_{1}, \cdots, \nu_{n}\right)
$$

if the following conditions are satisfied.

There exists a closed Riemann surface $\hat{S}$ of genus $g, h$ disjoint domains $\Delta_{1}, \cdots, \Delta_{h}$ and $n$ distinct points $p_{1}, \cdots, p_{n}$ on $\hat{S}$, and a conformal map $f$ of $U / G$ on to $S=\hat{S}-\left\{p_{l+1}, \cdots, p_{n}, \Delta_{1}, \cdots, \Delta_{h}\right\}$ such that $z \in U$ is not a fixed point of an elliptic element of $G$ if $f \circ \pi(z)$ $\neq p_{j}, 1 \leqq j \leqq l$, and is a fixed point of an elliptic element of order $\nu_{j}$ if $f \circ \pi(z)=p_{j}$. In this case we will say that the surface $S$ is of type $\left(g, h, n ; \nu_{1}, \cdots, \nu_{n}\right)$ and that $G$ represents $S$. Since (see [4]) $U / G$ is a surface of some type, every Fuchsian group has some signature.

We note that $h=0(h>0)$ is equivalent to the statement that $G$ is of the first (second) kind. Furthermore, by using Euler's formula and

Received by the editors, January 5, 1966.

1 Research sponsored by National Science Foundation grant GP-4079.

2 This follows from the work of Fenchel and Nielsen [5]. 
the formula for the sum of the angles of a hyperbolic polygon, it is easy to see that there are no Fuchsian groups having signature $(1,0,0)$.

We recall [1], [3], that a homeomorphic self map $w(z)$ of a domain $\Delta$ is quasiconformal if it has generalized derivatives in $\Delta$, satisfying a Beltrami equation $w_{\bar{z}}=\mu(z) w_{z}$ where $\mu(z)$ is measurable in $\Delta$ and $|\mu(z)| \leqq k<1$ for $z \in \Delta$. There exists in $U$ a unique solution $w^{\mu}(z)$ of the above Beltrami equation, satisfying $w(0)=0$ and $w(1)=1$. We call it the normalized $\mu$-conformal self map of $U$.

If $\mu$ depends continuously on a parameter, so does $w^{\mu}$. If $\mu$ is compatible with the Möbius transformation $A(z)$ (i.e., $\mu(A(z))$ $\left.=\left(A_{z} / A_{z}\right) \mu(z)\right)$, then $A^{\mu}=w^{\mu} \circ A \circ\left(w^{\mu}\right)^{-1}$ is a Möbius transformation. Furthermore, $w^{\mu}$ maps fixed points of $A$ into fixed points of $A^{\mu}$. $A^{\mu}$ is parabolic or elliptic of order $\nu$ if and only if $A$ is. If $G$ is a Fuchsian group generated by the transformations $\left\{A_{j}\right\}$, then the transformations $\left\{A_{j}^{\mu}\right\}$ generate a Fuchsian group $G^{\mu}$.

We may define quasiconformal maps of surfaces in terms of local coordinates. Then, the map $w^{\mu}$ of $U$ onto itself induces a quasiconformal map of $U / G$ onto $U / G^{\mu}$. If $S, S^{\prime}$, and $S^{\prime \prime}$ are three surfaces and $f^{\mu}, g^{\mu}$ quasiconformal maps of $S$ onto $S^{\prime}$ and $S^{\prime \prime}$, respectively, both satisfying the same Beltrami equation, then $g^{\mu} \circ\left(f^{\mu}\right)^{-1}$ is a conformal map of $S^{\prime}$ onto $S^{\prime \prime}$.

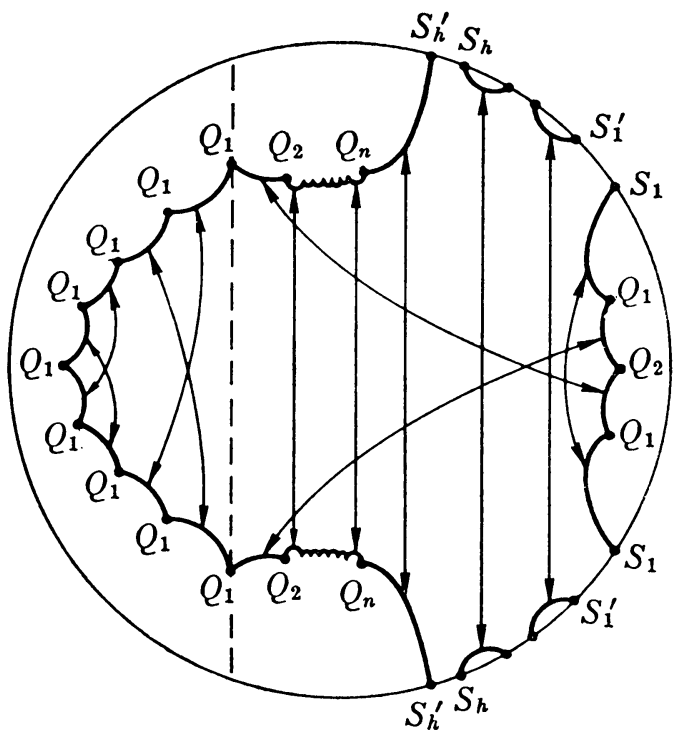

FIGURE 1 
2. Standard models. Given nonnegative integers $g, h$, and $n$ satisfying (1) but not

(a) $g=1, \quad h=0, \quad n=0$ or

(b) $g=0, h=0$ or 1 ,

we construct a standard group with signature $\left(g, h, n ; \nu_{1}, \cdots, \nu_{n}\right)$.

In the unit disc we construct a convex, noneuclidean polygon as shown in Figure 1.

The $Q_{j}$-vertices, $l+1 \leqq j \leqq n$, are on the unit circle, the sum of the angles at the $Q_{j}$-vertices, $1 \leqq j \leqq l$, is $2 \pi / \nu_{j}(2 \pi$ if $n=l=0)$.

The sides in the interior of $U$ are identified in pairs as shown in Figure 1 . In general, there will be $g-1$ collections of four sides (identified as shown) on the "left hand side" of the polygon, $n-1$ points $Q_{2}, \cdots, Q_{n}$ and $h$ "gaps" $\left(S_{1}, S_{1}^{\prime}\right), \cdots,\left(S_{h}, S_{h}^{\prime}\right)$ on the "right hand side."

We make the following clarifications and modifications:

(i) If $h=0$ and $n>1, S_{h}^{\prime}$ becomes $Q_{1}$.

(ii) If $h=0$ and $n=0$ or $1, S_{h}^{\prime}$ and $Q_{2}$ become $Q_{1}$.

(iii) If $h>0$ and $n=1, Q_{2}$ becomes $S_{h}^{\prime}$.

(iv) If $h>0$ and $n=0$, we choose the polygon as in Figure 2.

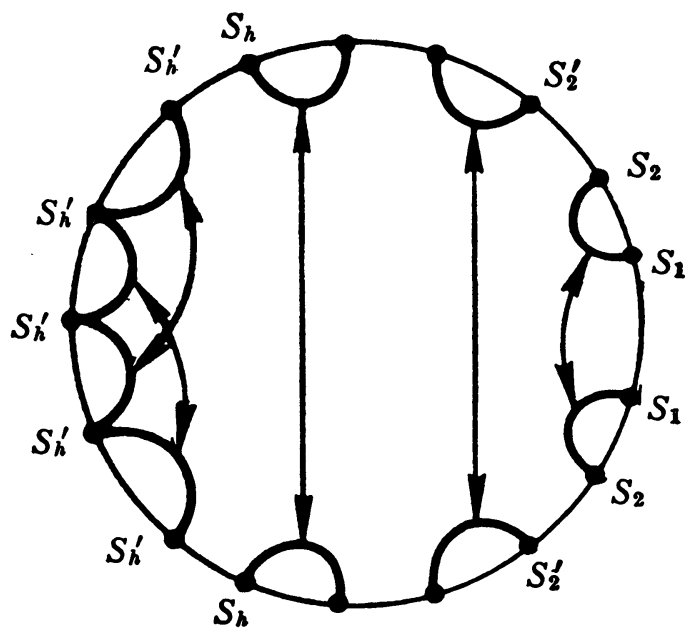

FIGURE 2

That such a polygon can be constructed (with the prescribed angles) in a canonical way may be shown by a simple continuity argument (see [6]). 
The identification of a pair of sides may be realized by a Möbius transformation (leaving $U$ fixed) carrying one side in to the other. For definiteness, if the two sides have end points on the unit circle, we choose the transformation: reflection in one side followed by reflection in the line of symmetry between the pair.

We have constructed a convex polygon whose sides are identified by a sequence $\left\{A_{j}\right\}$ of hyperbolic Möbius transformations. Let $G$ be the group generated by the $A_{j}$. Each set of $G$-equivalent vertices form a cycle. The sum of the angles of a cycle of interior vertices is $2 \pi / \nu$ where $\nu$ is a positive integer. Each vertex on the unit circle belongs to a cycle the sum of whose angles is 0 or $\pi$. (In the former case, although the generators of $G$ are hyperbolic, each vertex in the cycle is the fixed point of some parabolic element in $G$.) The polygon satisfies the hypothesis of Poincaré's theorem [2] and we may conclude that $G$ is a Fuchsian group with the polygon as a fundamental domain.

3. Deformation lemma. Let $G$ be a Fuchsian group with a signature $\left(g, h, n ; \nu_{1}, \cdots, \nu_{n}\right)$ having a (convex) fundamental polygon whose sides are identified in the same way as those of the polygon for the standard model with signature $\left(g, h, n: \nu_{1}, \cdots, \nu_{n}\right)$. Let $\mu(z)$ be defined and measurable in $U$ and satisfy $|\mu(z)| \leqq h<1, z \in U$. Suppose also that $\mu(z)$ is compatible with $G$. Let $w^{\mu}$ be the normalized $\mu$-conformal self map of $U$. Then $G^{\mu}$ is a Fuchsian group with signature $\left(g, h, n ; \nu_{1}, \cdots, \nu_{n}\right)$ and has a convex fundamental polygon whose sides are identified in the same way as those of the standard model.

The proof of this lemma is essentially contained in [6]. In particular, the case when $h+n=0$ may be proved as is Lemma $A_{3}$ of that paper. We wish, however, to give here the proof for $h+n>0$, divorced from symmetry considerations.

Proof. $(h+n>0)$. Consider the groups $G^{t \mu}, 0 \leqq t \leqq 1$ with $G^{0}=G$. Let $P(0)$ denote the fundamental polygon with vertices $\left\{V_{j}\right\}$ described in $\S 2$. Let $P(t)$ be the noneuclidean polygon obtained by joining the points $w^{t \mu}\left(V_{j}\right)$. Since, if two vertices of $P(0)$ are identified by $A \in G$, their images are identified by $A^{t \mu} \in G^{t \mu}$, the sides of $P(t)$ are identified in the same way as those of $P(0)$. The sum of the angles of any cycle of order $\nu$ remains $2 \pi / \nu$ and parabolic vertices are carried into parabolic vertices. Poincaré's theorem may therefore be used to insure that $P(1)=w^{\mu}(P(0))$ is a fundamental domain for the group $G^{\mu}$ if the interior of $P(1)$ is simply connected. We will show that it is convex. Let $T=\{t: 0 \leqq t \leqq 1, P(t)$ convex $\} . T$ is nonempty $(0 \in T)$ 
and open ( $w^{t \mu}$ depends continuously on $t$ ). That $T$ is closed follows essentially from the fact that each interior angle of $P(t)$ is always less than $\pi$ since every interior vertex is the fixed point of an elliptic transformation.

We conclude that $T$ is the entire interval so that $P(1)$ is convex and hence a fundamental polygon of the group $G^{\mu}$.

4. Hyperbolic generators. Suppose now that $F$ is a given Fuchsian group with signature $\left(g, h, n ; \nu_{1}, \cdots, \nu_{n}\right)$. As we have mentioned, $g, h$, and $n$ can never satisfy condition (2a). We assume here that (2b) is also not satisfied. Let $G_{S}$ be the standard group of $\$ 3$ with signature $\left(g, h, n ; \nu_{1}, \cdots, \nu_{n}\right)$. Then $U / G_{S}$ and $U / F$ are both Riemann surfaces of type $\left(g, h, n ; \nu_{1}, \cdots, \nu_{n}\right)$. There exists a homeomorphism $f$ of $U / G_{S}$ on to $U / F$, carrying the points corresponding to fixed points of elliptic or parabolic transformations of $G_{S}$ onto the points corresponding to fixed points of $F$, and the $h$ disjoint domains of $U / G_{S}$ onto the corresponding domains of $U / F$. We may assume [3] that $f$ is a quasiconformal homeomorphism. Then $f$ defines in $P_{S}$ (the fundamental polygon of $G_{S}$ ) a function $\mu(z)=f_{\bar{z}} / f_{z}$. We extend $\mu$ to $U$ by requiring that it be compatible with $G_{S}$, and observe that $\mu$ is then measurable and $|\mu(z)| \leqq h<1$ for $z \in U$. Let $w^{\mu}$ be the normalized $\mu$-conformal self map of $U$. Then $w^{\mu}$ induces a $\mu$-conformal map $\tilde{w}^{\mu}$ of $U / G_{S}$ onto $U / G_{S}^{\mu}$, so that $g=\tilde{w}^{\mu} \circ\left(f^{\mu}\right)^{-1}$ is a conformal map of $U / F$ onto $U / G_{S}^{\mu}$. Then $F=L \circ G_{S}^{\mu} \circ L^{-1}$ for some Möbius transformation $L$. But $G_{S}^{\mu}$ has, as a fundamental domain, a convex, noneuclidean polygon $P$, and $L(P)$, which is also a convex, noneuclidean polygon, is a fundamental domain of $F$.

We summarize these remarks in the

Theorem. Let $F$ be a Fuchsian group with signature $(g, h, n$; $\left.\nu_{1}, \cdots, \nu_{n}\right)$ where, if $g=0, h$ is neither 0 nor 1 . Then $F$ has, as a fundamental domain, a convex, noneuclidean polygon whose sides are identified by hyperbolic transformations.

We note the

COROLlaRY. Every Fuchsian group (with the above exceptions) may be generated by a system of hyperbolic transformations.

REMARK 1 . If $F$ is a Fuchsian group satisfying condition (2b), then $F$ does not have a fundamental polygon whose sides are identified by hyperbolic transformations.

Proof. Suppose such a polygon did exist. Let $s$ be the number of 
inequivalent in terior sides (i.e., the number of identifying transformations), $r$ the number of "sides" on the unit circle $(r=0$ if $h=0)$ and $k$ the number of cycles. It is easy to show, using Euler's formula, that $s=k-1$ if $h=0$, and $s=k-r$ if $h=1$. On the other hand, if every transformation identifying pairs of sides were hyperbolic, we would have $s \geqq k$.

REMARK 2. The result for $h+n=0$ is, of course, not new, and the "Fricke polygons" could also be used. For a surface with $h+n>0$ and a "canonical dissection," we observe that, if the quasiconformal $\operatorname{map} f$ (of $\S 4$ ) is chosen to be extremal, the construction of the polygon is canonical.

REMARK 3. Our choice of the form of the polygons was rather arbitrary and many other choices could have been made.

\section{REFERENCES}

1. L. V. Ahlfors and L. Bers, Riemann's mapping theorem for variable metrics, Ann. of Math. (2) 72 (1960), 385-404.

2. P. E. Appell and E. Goursat, Théorie des fonctions algébriques et de leurs intégrales, 2nd ed., Gauthier-Villars, Paris, 1929-1930.

3. L. Bers, Uniformization by Beltrami equations, Comm. Pure Appl. Math. 14 (1961), 215-228.

4. - A Atomorphic forms and Poincaré series for infinitely generated Fuchsian groups, Amer. J. Math. 87 (1965), 196-214.

5. W. Fenchel and J. Nielsen, Discontinuous groups of non-Euclidean motions, (to appear).

6. R. J. Sibner, Symmetric Fuchsian groups, (to appear).

STANFORd UNIVERSITY 